\title{
Reparación toracoscópica de atresia esofágica con y sin fistula traqueoesofágica
}

\author{
ISIDORA GARCÍA L. ${ }^{1,2}$, MARICARMEN OLIVOS P. ${ }^{1,2}$, \\ MARCELA SANTOS M. ${ }^{1,2}$, MIGUEL GUELFAND CH. ${ }^{1,2,3}$ \\ 1. Servicio de Cirugía Pediátrica, Hospital Dr. Exequiel González Cortés. \\ 2. Escuela de Medicina, Departamento de Pediatría y Cirugía Pediátrica, Universidad de Chile-Sede Sur. \\ 3. Clínica Las Condes.
}

\begin{abstract}
Thoracoscopic repair of esophageal atresia with and without tracheoesophageal fistula

Introduction: Esophageal atresia (EA) is the interruption of the continuity of the esophagus, with or without persistent communication with the trachea. Recent advances in surgical techniques have made possible correction with minimally invasive surgery (MIS). Objective: To evaluate the management of thoracoscopic technique in the treatment of EA. Patients and Method: Retrospective analysis of medical records in two centers was carried out between 2007 and 2012. Variables such as gestational age, gender, weight, type of esophageal atresia, malformations, surgery and postoperative complications were recorded. Results: Twenty patients, 15 of them with type III EA, 4 with type I and one unclassifiable, were part of the study. 13 patients underwent ligation, cut of tracheoesophageal fistula and end to end anastomosis. Two underwent laparoscopic gastrostomy and fistula ligation. One patient required conversion and underwent esophagostomy plus gastrostomy. 4 patients without fistula required laparoscopic gastrostomy and aspiration of the proximal esophagus to be able to perform esophageal anastomosis. In the postoperative period, pleural drainage and a transanastomotic feeding tube were installed. Seven patients required esophageal dilations and 4 patients developed stenosis due to reflux. Two had recurrent fistula, one with spontaneous resolution and another with endoscopic resolution. Three children died: two of them due to pathologies not related to surgery and one as a result of limitation of therapeutic effort after fistula patency. Discussion: Esophageal atresia repair with MIS is a safe and effective option with excellent exposure and visualization of anatomic landmarks and little associated morbidity.
\end{abstract}

(Key words: Esophageal atresia, tracheoesophageal fistula, thoracoscopy).

Rev Chil Pediatr 2014; 85 (4): 443-447

\section{RESUMEN}

Introducción: Atresia esofágica (AE) es la interrupción de la continuidad del esófago, con o sin comunicación con la tráquea. Los avances en cirugía han permitido su corrección con cirugía mínimamente invasiva (CMI). Objetivo: Evaluar el manejo toracoscópico en AE. Pacientes y Método: Revisión retrospectiva de fichas

Recibido el 21 de marzo de 2013, última versión aceptada para publicación el 18 de febrero de 2014.

Potenciales conflictos de interés: Este trabajo cumple con los requisitos sobre consentimiento /asentimiento informado, comité de ética, financiamiento, estudios animales y sobre la ausencia de conflictos de intereses según corresponda.

Correspondencia a:

Dr. Miguel Guelfand Ch.

E-mail: mguelfand@clc.cl 
de 2 centros entre 2007 y 2012. Se registraron variables como edad gestacional, sexo, peso, tipo de atresia esofágica, malformaciones, cirugías y complicaciones postquirúrgicas. Resultados: Veinte pacientes, $15 \mathrm{AE}$ tipo III, 4 tipo I y uno no clasificable. En 13 pacientes se realizó ligadura, sección de fístula tráqueo-esofágica y anastomosis término-terminal. Dos fueron sometidos a gastrostomía laparoscópica más ligadura de fístula. Un paciente requirió conversión y se realizó esofagostomía más gastrostomía. En 4 pacientes sin fístula, se realizó gastrostomía laparoscópica y aspiración del esófago proximal hasta lograr realizar la anastomosis esofágica. En el post-operatorio se instaló drenaje pleural y sonda trans-anastomótica. Siete pacientes requirieron dilataciones esofágicas y 4 desarrollaron estenosis por reflujo. Dos presentaron fístula recidivada, uno con resolución espontánea y el segundo de resolución endoscópica. Tres niños fallecieron: dos por patologías no relacionadas con la cirugía y uno con limitación de esfuerzo terapéutico con repermeabilización de fístula. Discusión: La atresia esofágica reparada con CMI es una opción segura y efectiva en esta serie, permitiendo excelente exposición y visualización de reparos anatómicos, con poca morbilidad asociada.

(Palabras clave: Atresia esofágica, fistula traqueoesofágica, toracoscopía).

Rev Chil Pediatr 2014; 85 (4): 443-447

\section{Introducción}

La Atresia esofágica (AE), con o sin fistula tráqueo-esofágica (FTE), es una anomalía congénita poco frecuente, la cual se presenta en 1 de cada 2.500 nacidos vivos ${ }^{1-2}$. La AE, en el $50 \%$ de los casos, se asocia a otras malformaciones congénitas, más frecuentemente con la asociación VACTERL y menos frecuente con la asociación $\mathrm{CHARGE}^{3}$. Su etiología es desconocida y el origen probablemente multifactorial. Puede sospecharse en el período antenatal por presencia de polihidroamnios, y en el período postnatal se asocia a síntomas como sialorrea, dificultad para alimentarse, distress respiratorio y episodios de cianosis. El diagnóstico se confirma al no ser posible pasar una sonda nasogástrica hasta el estómago, apoyado con una radiografía simple en la mayoría de los casos. El tratamiento definitivo incluye la resección de la fístula, cierre del defecto traqueal y la anastomosis primaria del esófago. En las atresias sin FTE (tipo "Long-Gap"), en que la separación entre ambos cabos esofágicos es muy amplia, se debe intentar un cierre primario diferido. Sólo en muy pocos casos es necesario realizar un reemplazo esofágico ${ }^{4-5}$.

Los avances en la cirugía mínimamente invasiva en recién nacidos han permitido a los cirujanos infantiles realizar estos procedimientos mediante estas técnicas, con resultados muy satisfactorios ${ }^{6}$. La rápida progresión y evolución de la cirugía mínimamente invasiva en la década de los 90, los avances en la tec- nología óptica, el instrumental endoscópico, y el aumento de las habilidades quirúrgicas, han llevado a una explosión en el uso de la toracoscopía para múltiples propósitos en niños ${ }^{7}$. El objetivo de este estudio es describir la utilidad y eficiencia de la toracoscopía en la corrección de varios tipos de atresia esofágica en recién nacidos y evaluar los resultados obtenidos con esta técnica.

\section{Pacientes y Método}

\section{Diseño del estudio}

Estudio descriptivo de pacientes operados de atresia de esófago. Se realizó una revisión retrospectiva de las fichas de todos los recién nacidos sometidos a reparación toracoscópica de la AE, desde enero de 2007 a mayo de 2012, en los Servicios de Cirugía Infantil del Hospital de Niños Exequiel González Cortés y Clínica Las Condes. Se analizaron variables como edad gestacional, sexo, peso al momento de la cirugía, tipo de atresia esofágica, malformaciones asociadas, cirugía realizada y complicaciones intra y post operatorias. Además se consignó el número de intervenciones por paciente, la necesidad de conversión a toracotomía, el número de pacientes que presentaron filtración de la anastomosis, el desarrollo de estenosis, pacientes que requirieron dilataciones esofágicas, y que desarrollaron reflujo gastroesofágico (RGE), requiriendo posteriormente una Fundoplicatura de Nissen como técnica de cirugía antirreflujo. 


\section{Técnica quirúrgica}

En todos los casos se utilizó la misma técnica, con el recién nacido en posición semiprono derecho $\left(60^{\circ}\right)$, para permitir que el pulmón caiga, alejándose del mediastino posterior. Para acceder a la cavidad torácica, en todos los pacientes, se utilizaron tres puertos: el primer trocar, de $5 \mathrm{~mm}$, se introdujo por debajo de la punta de la escápula, el segundo trocar, de 5 $\mathrm{mm}$, en la región axilar en el tercer espacio intercostal y el tercero de $3 \mathrm{~mm}$ se ubicó de acuerdo a la evaluación quirúrgica de los extremos del esófago, por lo general en el sexto o séptimo espacio intercostal. Se identificó la vena ácigos y se seccionó con coagulación monopolar (Hook). La fístula traqueoesofágica se ligó con 2 clips o ligadura, y fue seccionada con tijera. La anastomosis termino-terminal del esófago se realizó con sutura Ethibond ${ }^{\circledR}$, Prolene $^{\circledR}$ o PDS $^{\circledR} 4 / 0$, o 5/0 o 6/0 según el paciente, mediante puntos intra-corpóreos.

\section{Resultados}

Durante un período de 64 meses, 20 recién nacidos con atresia de esófago con y sin fístula traqueoesofágica se sometieron a reparación toracoscópica. Su edad gestacional media de nacimiento fue de 37 semanas (rango 32 a 38) y el peso promedio fue de 2,75 kilos (rango de $1,5$ a 5,2$)$.

Quince pacientes tenían atresia de esófago tipo III (fistula tráqueo esofágica de cabo esofágico distal), cuatro tenían atresias tipo I ( $\sin$ fístula, "Long Gap") y el restante un tipo de atresia no clasificada (doble fistula sin discontinuidad de esófago, con segmento no permeable).

En 13 pacientes se realizó ligadura y sección de fístula tráqueo-esofágica y anastomosis término terminal de los cabos esofágicos en forma primaria. Dos pacientes fueron sometidos a gastrostomía laparoscópica más ligadura de fistula como tratamiento inicial y en 4 pacientes con "Long Gap" fue necesario realizar gastrostomía con aspiración del esófago proximal, hasta lograr realizar anastomosis esofágica término terminal. Un paciente requirió conversión a toracotomía abierta y se le realizó una gastrostomía y esofagostomía (tabla 1).
De los 13 pacientes sometidos a anastomosis primario, 10 de ellos no tuvieron complicaciones. Un paciente presentó una filtración menor que se resolvió espontáneamente y otro presentó una fístula tráqueo-esofágica recidivada que requirió tratamiento endoscópico. Uno presentó neumotórax a repetición en el postoperatorio por problemas del drenaje torácico y 4 desarrollaron estenosis esofágica y RGE secundario (confirmando el diagnóstico con radiografia de esófago, estómago, duodeno y cuadro clínico), requiriendo una cirugía de Nissen laparoscópica y gastrostomía (tablas 2 y 3$)$.

Tabla 1. Clasificación de atresia esofágica

\begin{tabular}{|lc|}
\hline Tipo de atresia & n \\
\hline Tipo I & 4 \\
Tipo II & 0 \\
Tipo III & 15 \\
Tipo IV & 0 \\
Tipo V & 0 \\
\hline Inclasificable* & 1 \\
\hline
\end{tabular}

*Doble fistula sin discontinuidad de esófago, con segmento no permeable.

Tabla 2. Técnica quirúrgica empleada

\begin{tabular}{|lc|}
\hline Técnica quirúrgica & n \\
\hline Sección de Fístula y anastomosis primaria & 13 \\
\hline Sección de Fístula y Gastrostomía & 2 \\
\hline Gastrostomía y anastomosis termino terminal diferida & 4 \\
\hline Sección de Fístula, gastrostomia y esofagostomía & 1 \\
\hline
\end{tabular}

Tabla 3. Complicaciones post operatorias

\begin{tabular}{|lcll|}
\hline Complicaciones & Casos & Tratamiento \\
\hline Filtración de anastomosis & 1 & Resolución espontánea \\
\hline Recidiva de fístula & 1 & Tratamiento endoscópico \\
\hline Neumotórax a repetición* & 1 & Drenaje pleural \\
\hline Reflujo gastroesofágico & 4 & $\begin{array}{l}\text { Manejo médico (3 casos) } \\
\text { Operación de Nissen } \\
\text { (1 caso) }\end{array}$ \\
\hline
\end{tabular}

*Neumotórax secundario a disfunción de drenaje con sistema Acquaseal. 
En los 4 pacientes con Long Gap fue necesario medir los cabos bajo radioscopía cada 2 semanas hasta obtener una distancia entre los cabos no mayor a 2 cuerpos vertebrales. Todos fueron reconstituidos entre las 4 a 8 semanas posteriores. Tres pacientes se repararon por videotoracoscopía y en uno fue necesario convertir por tener los cabos muy distantes, por lo que se realizó gastrostomía más esofagostomía. De los 2 pacientes que se les realizó ligadura de fistula y gastrostomía como primera instancia, uno de ellos fue reconstituido 4 semanas después y el otro falleció en la espera, por patologías asociadas. A todos los pacientes sometidos a anastomosis termino-terminal se les instaló un drenaje pleural derecho y una sonda transanastomótica, logrando iniciar alimentación por sonda nasogástrica al $3^{\circ}$ ó $4^{\circ}$ día postoperatorio y al séptimo día se realizó un esofagograma previo a administrar alimentación vía oral.

Siete pacientes requirieron dilataciones esofágicas. Un paciente presentó traqueomalacia sintomática y estenosis esofágica, y un paciente presentó alteración de la motilidad esofágica. Tres pacientes fallecieron: en uno se re-permeabilizó la fístula y se limitó esfuerzo terapéutico por anomalías asociadas, los otros 2 por patologías no relacionadas con la cirugía. Hubo sólo 2 conversiones: una por condiciones del paciente y otra por distancia de los cabos. Actualmente los 17 pacientes se alimentan por boca sin problemas.

\section{Discusión}

Desde la aparición de la cirugía endoscópica, a fines de la década de los 80 , se ha progresado enormemente en las técnicas quirúrgicas mínimamente invasivas en recién nacidos y lactantes. Los avances recientes en los conocimientos de estas técnicas y del instrumental quirúrgico, han permitido ampliar el espectro de aplicaciones de la cirugía endoscópica durante los últimos años. Muchas de las intervenciones que clásicamente se realizaban por vía abierta, ahora pueden realizarse por vía mínimamente invasiva. Lo mismo ha ocurrido en la cirugía pediátrica. Procedimientos que se pensaba eran impracticables en niños, menos aún en recién nacidos, actualmente son parte de la práctica habitual de muchos centros pediátricos y se llevan a cabo a diario ${ }^{8}$. La videotoracoscopía en niños también ha tenido una evolución dramática desde que la primera atresia esofágica con fistula traqueo-esofágica fue reparada mediante esta técnica en 1990.

La reparación de la $\mathrm{AE}$ mediante técnicas mínimamente invasivas es muy beneficiosa, pero las dificultades técnicas son muchas. La mayor ventaja es que se puede evitar la realización de una toracotomía posterolateral en un recién nacido, lo que reduce las secuelas músculo-esqueléticas que a menudo se desarrollan después de esta cirugía ${ }^{8}$.

Se ha demostrado que este tipo de incisiones se asocia a múltiples complicaciones postoperatorias a largo plazo, entre la que destacan: escoliosis, escápula alada, asimetría de la pared torácica, elevación del hombro, trastornos del desarrollo del tejido muscular y tejidos blandos y debilidad de la cintura escapular. Es por esta morbilidad asociada que, durante los últimos años, se ha impulsado el desarrollo de técnicas mínimamente invasivas para el abordaje de esta patología ${ }^{1}$. Otro gran punto a favor es un resultado estético mucho mejor ${ }^{8}$.

Un beneficio inesperado de la toracoscopía es la mejor visualización de la anatomía de la cavidad torácica y, especialmente, de la fístula. La visión que se obtiene durante el procedimiento es excelente, lo que permite una mejor identificación de la fístula traqueoesofágica, una disección más precisa y la conservación de las estructuras vecinas más pequeñas, como las ramas del nervio vago en el esófago. Además, considerando que la fístula se visualiza perpendicular a su conexión con la tráquea membranosa, se puede identificar fácilmente el sitio exacto en que debe hacerse la ligadu$\mathrm{ra}^{1,7,8}$.

El abordaje transpleural necesario para la toracoscopía puede ser considerado una desventaja. Sin embargo, múltiples publicaciones han descrito pacientes intervenidos con este abordaje sin encontrar diferencias en la morbimortalidad con respecto al abordaje extrapleural. Por el contrario, se han publicado varios estudios no randomizados, en los que no se han podido establecer diferencias significativas en 
las tasas de morbilidad y mortalidad entre el abordaje extrapleural y el transpleural ${ }^{7-10}$.

El principal obstáculo técnico en esta cirugía es la sutura de la anastomosis. La colocación de las suturas y anudado son técnicamente exigentes. La sutura es compleja dado el limitado espacio de trabajo y el diámetro del esófago ${ }^{1,8}$.

En la evaluación de esta serie en curso, las características de los pacientes son muy similares a otras series clínicas mayores. No hubo complicaciones intraoperatorias, y todos los niños se recuperaron bien de la intervención siendo la incidencia de complicaciones postoperatorias similar ${ }^{11}$.

La asociación de reflujo gastroesofágico en pacientes con AE/FTE está bien documentada. Hay una tendencia reciente hacia una gestión más agresiva que resulta en la necesidad de menos fundoplicatura. La mayoría de los estudios contemporáneos han documentado una incidencia de fundoplicatura de $32,2 \%$ en estos pacientes ${ }^{7}$. En esta serie la tasa de fundoplicatura fue de un $20 \%$.

Las incisiones más pequeñas, estadías hospitalarias más cortas y el rápido retorno a las actividades preoperatorias hacen que la cirugía mínimamente invasiva siga siendo muy atractiva para los pacientes ${ }^{12,13}$. Existe poca literatura nacional e internacional que compare grupos operados por vía laparoscópica versus abierta, sin embargo, con los buenos resultados obtenidos por vía laparoscópica, por cirujano experimentado, no consideramos ético estudios prospectivos que realicen esta comparación.

En conclusion, la atresia esofágica reparada con cirugía mínimamente invasiva es una opción terapéutica segura y efectiva en esta serie, permitiendo una excelente exposición y visualización de los reparos anatómicos, facilitando la disección y con poca morbilidad asociada.

\section{Referencias}

1.- Rothenberg SS: Thoracoscopic repair of esophageal atresia and trachea-esophageal fistula. Semin Pediatr Surg 2005; 14: 2-7.

2.- Pierro A, Hall NJ, Chowdhury MM: Gastrointestinal surgery in the neonate. Current Paediatrics 2006; 16 : 153-64.

3.- El-Gohary Y, Gittes GK, Tovar JA: Congenital anomalies of the esophagus. Semin Pediatr Surg 2010; 19: 186-93.

4.- Hall NJ, Eaton S, Pierro A: The evidence base for neonatal surgery. Early Human development 2009; 85: 713-8.

5.- Arul GS, Parikh D: Oesophageal replacement in children. Ann R Coll Surg Engl 2008; 90: 7-12.

6.- Rothenberg SS: Thoracoscopic repair of esophageal atresia and tracheo-esophageal fistula in neonates: evolution of a technique. J Laparoendosc Adv Surg Tech A 2012; 22: 195-9.

7.- Holcomb GW, Rothenberg SS, Bax KM, et al: Thoracoscopic repair of esophageal atresia and tracheoesophageal fistula: a multi-institutional analysis. Ann Surg 2005; 242: 422-8.

8.- Rothenberg SS: Thoracoscopic repair of tracheoesophageal fistula in newborns. J Pediatr Surg 2002; 37: 86972.

9.- Shah A, Shah A: Minimally Invasive Surgery. Indian J Pediatr 2008; 75: 925-9.

10.- Tsao K, Lee H: Extrapleural thoracoscopic repair of esophageal atresia with traqueoesophageal fistula. Pediatr Surg Int 2005; 21: 308-10.

11.- Jakubson L, Paz F, Zavala A, Harris P, Bertrand P: Atresia Esofágica y fístula traqueoesofágica. Evolución y complicaciones postquirúrgicas. Rev Chil Pediatr 2010; 81: 339-46.

12.- Zitsman JL: Pediatric Minimal-Access Surgery: Update 2006. Pediatrics 2006; 118: 304-8.

13.- Guelfand M: Patología quirúrgica neonatal: orientación diagnóstica y aportes de la cirugía mínimamente invasiva. Rev Med Clin Condes 2009; 20: 745-57. 